\title{
Fehlen der aufsteigenden Aorta, fast vollständiges Fehlen des Septum ventriculorum und des linken Ventrikels bei einem Neugeborenen, welcher trotz- dem über vier Tage gelebt hat.
}

\author{
Von \\ Dr. Robert Ziegenspeck, \\ Privatdocent an der Universität, Assistent des Herrn Gehoimen Medicinalrath
}

Prof. Dr. Winckel in München.

(Mit 4. Abbildungen auf Tafe1 I.)

Am 1. Juli 1887 wurde von der ledigen Köchin Marie K. in der königlichen Universitäts-Frauenklinik hierselbst ein Knabe geboren, welcher am fünften Tage nach der Geburt starb und. dessen Herz durch die Art der daran befindlichen Verbildungen, sowie durch deren Einfluss auf den fötalen wie extrauterinen Kreislauf in hohem Grade unser Interesse in Anspruch nimmt.

Die K. war 28 Jahre alt und zum ersten Male schwanger. Sie war erst seit ihrem 20. Jahre menstruirt, im 20. und dann im 22. Jahre wegen Amenorrhoë behandelt worden und hatte auch in der Zwischenzeit nur äusserst spärliche, zwei- bis dreitägige, höchst schmerzhafte Menses.

Die letzte Menstruation hatte anfangs September vorigen Jahres stattgefunden. Das Kind wäre demnach einige Wochen über die Zeit getragen worden.

Die Geburt erfolgte aus I. Schädellage nach zwölfstündiger Eröffnungs- und zweistündiger Austreibungsperiode spontan. Nachgeburt wurde zwei Stunden später nach Credé entwickelt. Das Wochenbett verlief ohne Störung, bis auf Schmerzhaftigkeit der Brustwarzen, welche insofern hervorgehoben $\mathrm{zu}$ werden verdient, als es darauf schliessen lässt, dass das Kind mit Energie gesaugt haben muss.

Dass die Herzaction vor der Geburt durch die Verbildung nicht beeinträchtigt worden war, dafür spricht der Umstand, dass 
136 Doppelschläge sehr kräftig in normalem Rhythmus gezählt worden sind.

Das Gewicht des Kindes betrug $3150 \mathrm{~g}$; die Länge $50 \mathrm{~cm}$. Auch die Schädelmaasse entsprachen einem zwar nicht übermässig grossen, aber ausgetragenen Kinde.

Es betrug:

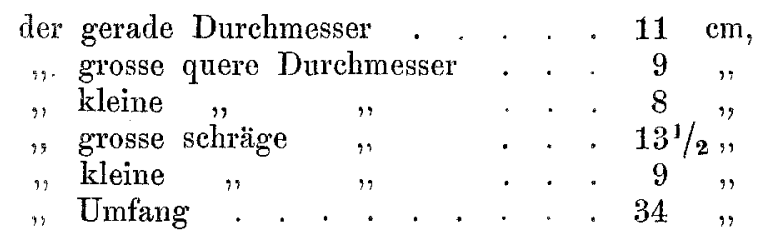

Im Uebrigen zeigte der Knabe alle Zeichen der Reife. Er bot durchaus nichts von der Norm Abweichendes dar. Höchstens wäre ein etwas „marmorirtes" Aussehen der Haut zu nennen, das gleich in den ersten Tagen bemerkt wurde, und welches allmälig in Cyanose ïberging. Die Stimme war stets kräftig und klar. Vom vierten Tage an verfiel das Kind mehr und mehr in Schlafsucht. Erst am vierten Tage fällt es auf, dass es einen ausgeprägten Icterus zeigt, keine Nahrung mehr zu sich nimmt und bald mehr, bald weniger blau wird. Defäcation und Exurese sind dabei ungestört.

Am fünften Tage, dem 5. Juli, erfolgt der Tod, ohne dass Erstickumgsanfälle vorhergegangen wären; das Kind schlief ein und erwachte nicht wieder.

Section, ausgeführt von Herrn Professor Bollinger am 6. Juli 1887, Nr., 302: Länge 51,5 cm, Gewicht $2790 \mathrm{~g}$, Gehirn $39 \mathrm{~g}$, Lunge je $44 \mathrm{~g}$, Herz $29 \mathrm{~g}$, Leber $95 \mathrm{~g}$, Milz $15 \mathrm{~g}$, Niere je $25 \mathrm{~g}$.

Haut schmutzig gelbgrau, Bauchdecken grünlich verfärbt, Nabelschnurinsertionsstelle scharf abgesetzt, kunstgerecht unterbunden, Haare ziemlich reichlich, 2-3 cm lang, Fontanelle sehr klein.

Kopf: Umfang $36 \mathrm{~cm}$.

\begin{tabular}{|c|c|}
\hline emporaldurchmesser & 8 \\
\hline Biparietaldurchmesser & 10 \\
\hline Fronto-occipitaldurchmesser & 11 \\
\hline italdurehmesser. & 13,5 \\
\hline aboccipito-frontaldurchmesser & 11 \\
\hline
\end{tabular}

A ugen geschlossen, Conjunctivae leicht icterisch, Pupillen erweitert. 
Nägel etwas livid, ragen bis an die Fingerkuppen. Querdurchmesser an der Schulter 12,5, an den Hüften $10 \mathrm{~cm}$. Sagittaldurchmesser des Brustkorbes $9,8 \mathrm{~cm}$.

Hod en im Hodensacke. Lanugo spärlich, Fettpolster mässig, Muskulatur mässig kräftig, Todtenstarre gelöst. An der unteren Epiphyse des Femur Knochenkern deutlich, linsengross.

Bauchhöhle: Muskulatur trüb, grau-bräunlich, Zwerchfell rechts am vierten Intercostalraum, links in der Höhe der vierten Rippe. Leber vor dem Magen fingerbreit im Umfange von einem halben Hühnerei vorliegend.

Brusthöhle: Herz liegt zwei- bis dreimarkstückgross vor den Lungen, dieselben unten stark, oben wenig retrahirt. Herzbeutel fast leer. Thymusdrüse vom Umfange einer kleinen Pflaume. Im linken Pleurasacke einige Tropfen Serum, rechts ebenso.

Nervensystem: Besonders über dem rechten Os parietale das subcutane Gewebe ödematös, blutdurchsetzt. Grosse Fontanelle sehr klein. Sinus longitudinalis enthält reichlich dunkle Gerinnsel. Basis gehörig. Anämie daselbst noch grösser. Gehirn schneidet sich weich. Schnittfläche stark glänzend, Blutung spärlich, leicht röthlicher Ton. Graue und weisse Substanz nicht geschieden, Seitenventrikel erweitert, besonders die Hinterhörner. Adergeflechte dunkel blutreich.

Respirationsorgane. Mundhöhle ohne abnormen Inhalt, Schleimhaut blass. Zunge blass, in der Rachenhöhle etwas schleimiger Beschlag. Oesophagus vollständig leer. Schleimhaut des Rachens etwas geröthet, am Kehlkopfeingange blass. Im Kehlk opf und Trachea fast kein Inhalt, Schleimhaut blass, Bronchien enthalten etwas weisslichen Schaum. Schilddrüsen pflaumenkerngross, etwas blutreich. Lungen von mässigem Umfange, hühnereigross, vorn oben hell ziegelroth, lobuläre Zeichnung deutlich, hinten unten dunkel livid. Pleura glatt, durchsichtig glänzend. Subpleurale Ecchymosen; an einzelnen Stellen erstrecken sich dieselben in die Randpartien des Gewebes, vorn oben weich, hinten unten etwas derber. Beim Einschneiden leichtes Knistern. Oben vorn mässig blutreich, hinten unten blutreicher, dunkelbraunroth mit einem Stich ins bläuliche. Lufthaltige, schaumige Flüssigkeit abstreifbar. Lunge und Herz schwimmen, sämmtliche einzelnen Theile der Lunge schwimmen auch. 
Harnapparat: Nierenkapsel leicht abziehbar. Am Hilus der linken Niere eine blutige Suggillation von geringem Umfange, deutliche Lappung, fleckig graue Farbe. Grenze zwischen Rinde und Mark deutlich. Radiäre goldgelbe Streifung der Marksubstanz: Harnsäureinfarct.

Circulationsapparat: Milz 5,5:3:2 cm. Kapsel durchsichtig, Farbe braunlivid, Consistenz ziemlich derb. Rechter Vorhof mässig gefüllt, haselnussgross, linker fast collabirt. Im rechten Vorhofe dunkelschwarzes, dickflüssiges Blut, 1/2 Esslöffel voll. Im rechten Ventrikel 1/4 Theelöffel voll gleichen Blutes. Im linken Vorhofe einige Tropfen Blut, ebenso im linken Ventrikel.

Rechter Ventrikel: Wandung auffallend dick, sehr weit.

Linker Ventrikel sehr eng, dünn. Dicke des rechten Ventrikels 0,6 cm. Lungenarterie sehr weit. Ductus Botallii in Verbindung mit Aorta (descendens). Aorta ascendens fehlt (an deren Stelle ein dünner Bindegewebsstrang). Linker Ventrikel sehr eng, Wandung $0,3-0,4 \mathrm{~cm}$ dick, communicirt durch eine Oeffinung des Septum ventriculorum mit dem rechten Ventrikel; die Oeffnung ist für einen kleinen Finger durchgängig.

Soweit das Sectionsprotokoll.

Das Herz wurde uns von Herrn Professor Bollinger gütigst überlassen, und Herr Geheimrath Prof. Winckel ertheilte mir den Auftrag, dasselbe zu veröffentlichen; ich sage ihm dafür meinen besten Dank.

Statt weitläufiger Beschreibung der schon oben im Sectionsprotokolle genügend erwähnten Verbildungen habe ich in Fig. 2, 3 und 4, Taf. I, je eine Ansicht des betreffenden Präparates von vorn, von hinten und von unten gezeichnet und hoffe, dass diese Zeichnungen genügen, dem Leser ein deutliches Bild derselben zu verschaffen. Damit man bequem das Krankhafte mit dem Gesunden vergleichen könne, habe ich in Fig. 1, Taf. I, das normale Herz eines neugeborenen Knaben wiedergegeben, dessen Gefässsystem ich vor einigen Jahren in Jena mit Gelatinelösung von der Nabelvene aus injicirt, präparirt und gezeichnet hatte. Das Herz mit seinen Gefässen ist in einer Ansicht von vorn dargestellt. Da es von einem über $4 \mathrm{~kg}$ schweren Kinde stammt und ausserdem mit Leimmasse prall gefültt war, ist es in allen Durchmessern grösser als dasjenige des Kindes K. Sämmtliche vier Abbildungen sind in natïrlicher Grösse nach Messungen angefertigt. Ueber alle Einzelheiten geben Bezeichnungen Aufschluss, welche an den Figuren angebracht sind. 
Die Aorta descendens ist mit einem Doppelhäkchen nach oben angezogen, die vom Arcus aortae entspringenden Gefässe sind nach rechts umgelegt, damit auch diejenigen Gefässe des Fötalkreislaufes sichtbar werden, welche sonst hinter dem Herzen liegen. Ich hoffe, dass die Zeichnungen dadurch nicht an Uebersichtlichkeit verlieren.

Da man vor der Section eine derartige Verbildung des Herzens nicht ahnen konnte, so wurde dasselbe ohne besondere Vorsicht wie jedes andere Herz herausgenommen. Dabei entstanden an demselben verschiedene Defecte, welche ich in den Zeichnungen mittels punktirter Contouren nach bestem Ermessen ergänzt habe.

In Fig. 4 sieht man, dass die untere Hohlvene mit dem grössten Theile der hinteren (eigentlich unteren) Vorhofswand weggeschnitten ist. Dadurch ist der rechte, beträchtlich dilatirte Vorhof breit geöffnet. Man erblickt in demselben nach unten das Ostium venosum dextrum. Dasselbe ist nicht über die Norm weit, jedenfalls schlussfähig. Oben im Bilde befindet sich die Einmündung der oberen Hohlvene, ganz links die Einmündung der Vena magna cordis. Der kleine und unbedeutende linke Vorhof ist durch eine Membran vollständig abgeschlossen, welche nur von ihrem vorderen (eigentlich oberen) Ende eine spaltförmige Oeffnung aufweist, durch welche das Blut der Lungenvenen, das zum linken Vorhof fliesst, in den rechten Vorhof und weiter in den rechten Ventrikel befördert wurde. Das Ostium venosum sinistrum ist nämlich vollständig verschlossen. Mit den feinsten Sonden gelangt man weder vom linken Vorhofe aus in den rudimentären linken Ventrikel, noch von diesem aus in den ebenfalls rudimentären linken Vorhof. Die Lungenvenen zeigen dagegen eine der Norm entsprechende Ausbildung. In Fig. 3 sieht man den unvollständig ausgebildeten linken Ventrikel geöffnet und das verkümmerte Herzohr, sowie die linke (eigentlich hintere) Wand desselben nach oben geschlagen. Man bemerkt, dass hier die Wandung beträchtlich dünner ist, als anderswo an dem gemeinsamen Ventrikel. Auch gewahrt man einige spärliche Trabekeln an jener Stelle, wo sonst das Septum ventriculorum sich befindet. Dieselben bilden aber nirgends eine zusammenhängende Scheidewand.

Auch in Fig. 2 sieht man das verkümmerte linke Herzohr und die nach oben umgeschlagene Wandung des linken Ventrikels. Das Herz ist in einer Ansicht von vorn dargestellt, wie das 
Taf. I.

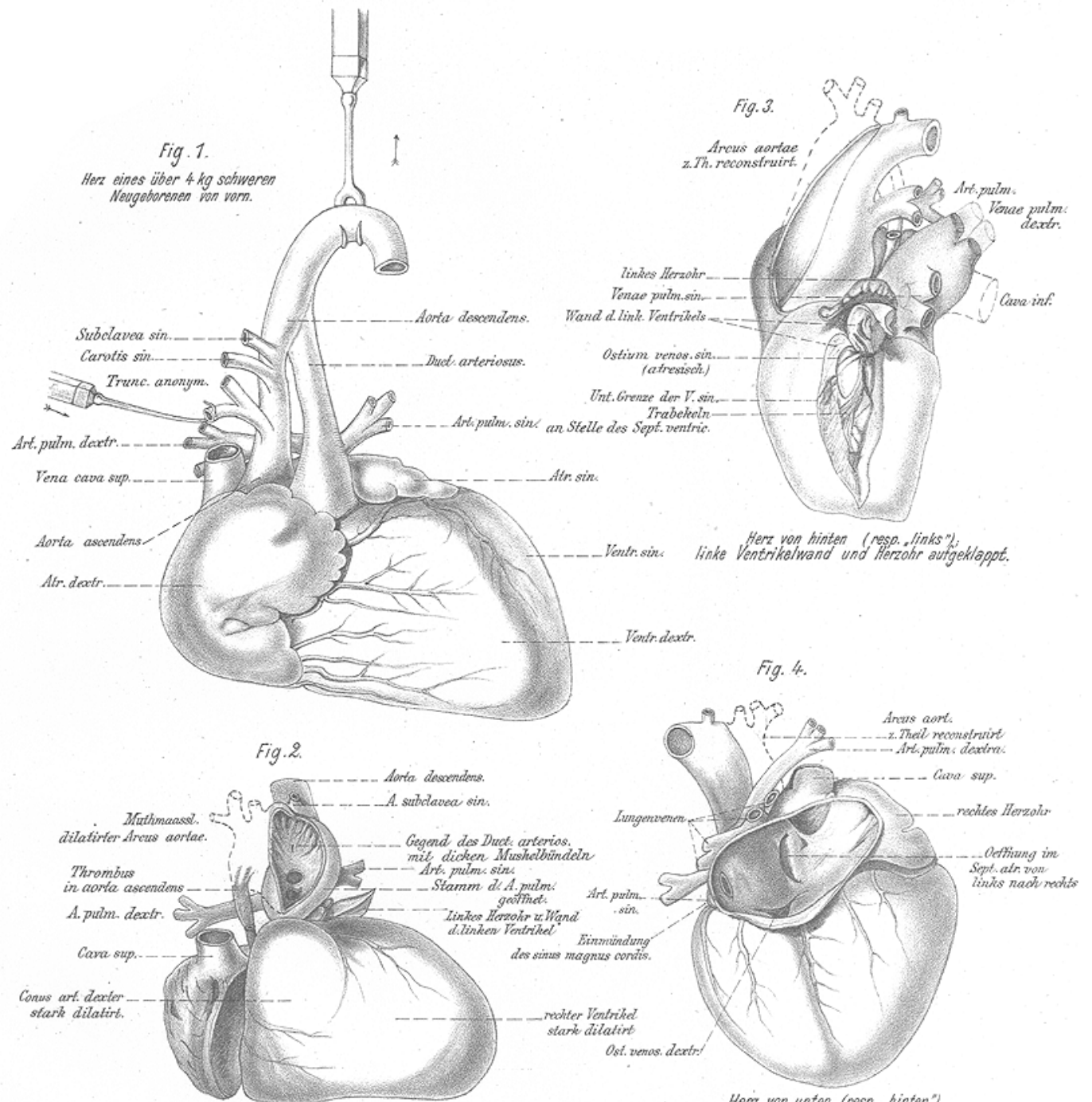

Herz von unten (rese, ,hinten"),

Herz von vorn (resa, rechts")

das Aneurysma des Stammes der Lungenarterie vervol/ständigt.

Vena cava inf. und untere Vorhofswand entfernt.

Archiv f. Gynổkologie Bd.XXXII.

tiathast EAfurbe,teipzig. 
in Fig. 1 abgebildete normale. Daher ist hier vor Allem ein Vergleich von Nutzen. Der leere rechte Vorhof des verbildeten Herzens ist schlaff und retrahirt, wodurch die mächtige Entwickelung des rechten Conus arteriosus so recht sichtbar wird; der des gesunden ist von der Injectionsmasse prall gefüllt, ausgedehnt und bedeckt zum grossen Theil den Conus arteriosus. Aus dem veränderten Verlaufe der Gefässe, welche aus den Kranzgefässen des kranken Herzens stammen, ersieht man leicht, wie sich derselbe so gleichsam zwischen dieselben gedrängt und sie verzerrt hat. Die bedeutendsten Vexänderungen zeigt indessen der Stamm der Lungenarterie.

Derselbe ist im ganzen Verlaufe aneurysmatisch ausgedehnt und seine Wandung namentlich in seinem rom Herzen abgewendeten Ende von trabekelartig vorspringenden Muskelbalken verstärkt. Da, wo sonst der Ductus arteriosus in die Aorta einmündet, befindet sich die Arteria subclavea sinistra. Leider ist gerade da, wo die übrigen Gefässe des Arcus aortae aus dem Aneurysma der Lungenarterie entsprungen wären, die Wandung desselben bei der Section verloren gegangen. Nur die halbe Peripherie des Ursprungs der Carotis sinistra ist noch vorhanden.

Ich habe nun zum Zwecke grösserer Anschaulichkeit den Stamm der Arterie mit punktirtem Contour ergänzt und ebenso die Arteria anonyma mit der Wand des Aneurysma so wiederhergestellt, wie sie höchst wahrscheinlich beschaffen war.

Als ich nun nach Resten von der Aorta ascendens suchte, fand sich rechts von dem Aneurysma der Lungenarterie eine graue Masse wie narbiges Bindegewebe und im Innern derselben ein roth gefärbtes Gewebe, dem Anscheine nach ein bindegewebig organisirter Thrombus. Als ich diese Masse mit der Scheere abschnitt, fand ich am unteren Ende ein leeres Gefäss, welches sich in derselben verlor und dessen Lumen für eine dünne Sonde bequem zugängig war. Man gelangte durch dasselbe herzwärts in einen flachen, blind endigenden Raum, den Rest des Conus arteriosus aortae. Vom rudimentären linken Ventrikel aus war es unmöglich, in denselben zu gelangen. Somit lag für das Zustandekommen der Verbildung die Erklärung ziemlich nahe.

$\mathrm{Zu}$ einer Zeit des intrauterinen Lebens, wo das ganze Herz ungefähr die Ausdehnung des jetzigen linken Ventrikels nebst Herzohr zeigte und wo das Septum ventriculorum noch nicht geschlossen war, führten entzündliche Processe am Ostium venosum 
sinistrum einestheils zu Stenose und Verschluss desselben, anderentheils zur Bildung von Thromben und zu Thrombose der aufsteigenden Aorta. Die linke Herzhälfte war somit ausser Thätigkeit und verkümmerte, die rechte hypertrophirte compensirend. Das Blut, welches die Lungenvenen dem linken Vorhofe zuführen, wurde vom rechten Ventrikel aspirirt und bahnte sich nach rechts einen Weg, der sonst nicht besteht. Zur Bildung eines regulären Septum atriorum kam es nicht. Es scheint überhaupt zu dessen Entwickelung die zwischen die Vorhöfe hinein vorspringende Aorta nothwendig zu sein.

Die Gefässe des Arcus aortae aber, welche ja den Kopf und die Arme zu versorgen haben, sowie die Aorta descendens mussten von der Lungenarterie aus gefüllt werden. Demnach musste an der Einmündungsstelle des Ductus arteriosus der Blutstrom sich theilen und der eine Theil des Blutes zurücklaufen, um den Arcus aortae zu füllen. Infolge davon entstanden Wirbelströmungen, Dilatation der betroffenen Arterienabschnitte, Hypertrophie der Muskulatur ihrer Wandung und fortschreitende Abstumpfung des Winkels, unter welchem die beiden Gefässe ursprünglich in einander einmündeten.

Soviel zur Erklärung der Zeichnungen und zur Ergänzung des Sectionsprotokolls. Diese Missbildung des Herzens ist gewiss sehr selten, vielleicht einzig in ihrer Art. Sie ist jedoch nicht allein durch ihre Seltenheit interessant, sondern wir erhalten durch sie wichtige Aufschlüsse über den Kreislauf des Ungeborenen.

Wenn wir die Lehrbücher der Geburtshülfe, auch die neuesten, und die Lehrbücher der Physiologie in Bezug auf den Fötalkreislauf befragen, so begegnen wir fast überall noch dem Kreislaufe in Form einer 8.

Diese Lehre wurde von Sabatier ${ }^{1}$ ) zuerst aufgestellt. Offenbar gelangte er zu dieser Anschauung, weil er glaubte, dass beim Ungeborenen der Kreislauf des venösen, d. h. sauerstoffarmen Blutes von dem des arteriellen, sauerstoffreichen, ebenso getrennt sei, wie beim Geborenen. Es soll sich die untere Hohlvene, welche das sauerstoffreiche, mit Nährstoffen beladene Blut der Nabelvene enthält und nur eine relativ geringe Menge venösen

1) Hist. de l'académie des sciences 1774, p. 7 et 198: Traité complet d'anatomie. Paris 1779. T. III, p. 386. Ref. in Knabbe, Disquisitiones historico-criticae. Diss, inaug. Bonn 1834. 
Blutes aus dem unteren Körper bekommt, vollständig in den linken Vorhof ergiessen und ebenso vollständig durch den linken Ventrikel in den oberen Körper befördert werden, so dass aus dem Arcus aortae dem unteren Körper durch die Aorta descendens gar nichts zugeführt werden sollte. In der oberen Hohlvene gesammelt, sollte dann dasselbe Blut, jetzt leicht venös, in den rechten Vorhof sich ergiessen. Dies war der obere Bogen der 8. Von hier sollte es nun mit Vernachlässigung des Lungenkreislaufes durch den Ductus arteriosus ${ }^{1}$ ) der Aorta descendens zugeführt werden, um dann theils in den unteren Körper, theils in die Placenta zu fliessen, von wo es in der unteren Hohlvene gesammelt dem linken Herzen wieder zufliessen sollte. Dies war der untere Bogen der 8, und das Blut der beiden Herzhälften sollte sich entschieden nirgends im Körper vermischen.

Mit der Anschauung, dass auf diese Weise die vordere (obere) Körperhälfte vorzugsweise arterielles, nährstoffreiches Blut erhalte, fand man die überwiegende Ausbildung dieser Regionen des Körpers beim Fötus in auffallender Uebereinstimmung.

Dieser Anschauung entgegen stand die ältere Lehre, welche von $\mathrm{Harve}^{2}$ ), dem Entdecker des Kreislaufes, selbst stammte, und nach welcher das ganze Blut beider Hohlvenen im rechten Vorhofe sich vermischen sollte. Dieses Gemenge des Blutes beider Hohlvenen wird theilweise durch den rechten Ventrikel in die Lungen und in die Aorta descendens befördert, zum Theil sollte es aber auch durch das eiförmige Loch in der Vorhofsscheidewand in den linken Vorhof gelangen, um mit dem Blute der Lungenvenen vermengt die obere Körperhälfte zu versorgen. Harvey betont ausdrïcklich, dass sich das Blut der beiden Herzhälften an mehreren Stellen im Körper vermische, und vergleicht den Fötus in Bezug auf den Kreislauf einem Fische. Letzterer besitzt nur einen Vorhof und eine Herzkammer, der Fötus benutzt vermittelst jener, seinem Kreislaufe eigenthümlichen Wege seine beiden Herzhälften wie ein einkammeriges Herz.

Wenn nun auch die Lehre Harvey's nicht ganz den That-

1) Dieser Gang wird mit Unrecht nach Botallius genannt, da er nach Kuabbe schon dem Galen bekannt war und von ihm als Ductus novus erwähnt wurde.

2) Harvey, De motu cordis et sanguinis etc. L. B., p. 162. 
sachen entspricht, wenngleich von $\mathrm{T} \mathrm{rew}^{1}$ ), Roedere $\mathrm{r}^{2}$ ), Halle $\mathrm{r}^{3}$ ), Senac ${ }^{4}$ ), Wolff ${ }^{5}$ ), Kilian ${ }^{6}$ ), Rüdinger ${ }^{7}$ ) and $\operatorname{mir}^{8}$ ) der anatomische Nachweis geführt worden ist, dass nicht ein Gemenge des Blutes beider Hohlvenen, sondern nur ein Theil des Blutes der unteren Hohlvene durch das Foramen ovale in die linke Herzhälfte fliessen kann, so bleibt doch dies Eine über allem Zweifel erhaben, dass Vermischungen des Blutes beider Herzhälften im Fötalkreislaufe stattfinden, und dass der Fötus zwei Herzkammern wie eine einzige benutzt.

Die Richtigkeit des letzteren Satzes wird nun durch den oben beschriebenen Fall vollkommen bestätigt. Der Fötus hatte nur eine Herzkammer zur Verfügung, welche allerdings mit der Function auch die Grösse zweier Herzkammern angenommen hatte. Trotzdem nun die vordere Körperhälfte nicht durch sauerstoffreicheres und nährstoffreicheres Blut bevorzugt worden sein $\mathrm{kan} n$, so hat sie dennoch eine Ausbildung gezeigt, welche in nichts hinter dem Grade der Entwickelung zurückblieb, welchen der ganze übrige Körper des Kindes zeigte. Schädelmaasse und Gewicht der einzelnen Organe des vorderen Körpers entsprechen in jeder Hinsicht der Länge und dem Gewichte des Kindes. Dabei giebt es genügende Anhaltspunkte, welche darauf hinweisen, dass die Verbildung ziemlich früh im Fötalleben eingetreten ist.

Während die anscheinend so frühzeitig eingetretenen Verkümmerungen eines Theiles der Blutwege durchaus nicht hin-

1) Diss. epist. de different. quibusdam inter natum et nascendum intercedentibus. Novembris 1736 . p. 41 (Knabbe).

2) De foetu perfecto. Argent. 1750, in Halleri disput. anat. Tom. VII, pr. II, p. 313 (Knabbe).

3) Elementa physiologiae corporis humani. Bern 1766. Tom. VIII, sect. IV, p. 373, et opera min. Tom. I, 39.

4) Traité de la structure du coeur, de son action et de ses maladies. Paris 1777. Tom. I, p. 259.

5) De foramine ovali ejusque in dirigendo sanguinis motu observationes novae in novae commentarii scient. Petropol. 1775. Tom. XX, p. 357.

6) Ueber den Kreislauf des Kindes, welches noch nicht geathmet hat. Karlsruhe 1826.

7) Ueber die Topographie der beiden Vorhöfe u. s. w. Journ. f. Kinderkrankheiten 1871, Bd. XXIX, S. 402, und Topograph.-chirurg. Anatomie des Menschen. Stuttgart 1873. Taf. XIII.

8) Welche Veränderungen erfährt die fötale Herzthätigkeit regelmässig durch die Geburt. Gekr. Preisarbeit. Inaug.-Diss. Jena 1882, und in Preyer's Physiologie des Embryo, Anhang: Ueber den Blutkreislauf des Säugethierund Menschen-Foetus. Jena 1884. 
derten, dass das Kind zur vollen Reife sich entwickelte, wurden sie sehr bald verderblich, als der Lungenkreislauf in Gang kommen sollte. Mit jeder Inspiration musste eine beträchtliche negative Schwankung im arteriellen Blutdruck eintreten, während bei der Exspiration ein Theil des Blutes, ohne in den Lungen regenerirt zu sein, der Aorta descendens zuströmte. Da die Lungen bei der inspiratorischen Erweiterung des Thorax ihr Blut aus derjenigen Herzhälfte aspirirten, welche zugleich dem grossen Körperkreislaufe gedient hat, so ist anzunehmen, dass überhaupt der Druck im Arteriensysteme des grossen Körperkreislaufes nicht so gross geworden ist, als wenn er wie sonst von einem besonderen Ventrikel erzeugt worden wäre und seine Arterien von den Lungen abgeschlossen gewesen wären. Aus diesen Gründen mag wohl der Druck im Venensystem des Körpers oft geringer gewesen sein als in den Gallengängen der Leber, so dass ein Uebertritt der Galle in die Lebervenen stattfinden konnte. Ein beträchtlicher Icterus trat daher auf, bevor eine ausgesprochene Cyanose sich einstellte, welche letztere dann schliesslich, langsam und allmälig auftretend, ohne Erstickungszufälle den Tod herbeiführte.

Der Fall ist noch sofern von Interesse, als er eine geistreiche Beweisführung Kilian's erschüttert, welche dieser zu Gunsten der Anschauung geltend gemacht hatte, dass die untere Hohlvene in beide Vorhöfe gleichzeitig ihr Blut ergiesse und niemals Blut aus dem rechten Vorhofe in den linken übertrete. Nach Beschreibung anatomischer Verhältnisse, welche diese Anschauung zu stützen genügt hätten, fügt er fragend hinzu; „Wann soll denn das Blut aus dem rechten Vorhofe in den linken fliessen?! in der Systole oder in der Diastole? Es müsste ja dann der rechte Vorhof in Systole sein, während der linke in Diastole ist und umgekehrt, und das widerspricht den Thatsachen."

Das oben beschriebene Präparat zeigt uns, dass diese indirecte Beweisführung nicht genügen könnte, wenn keine bessere directe vorhanden wäre. Ebenso, wie hier die Aspirationskraft des erschlaffenden rechten Ventrikels im Beginn der Diastole im Stande war, auch bei gleichzeitiger Systole beider Vorhöfe den Druck rechts so zu vermindern, dass ein Ueberströmen von links nach rechts und in den Ventrikel hinein stattfinden konnte, ebenso würde der linke Ventrikel beim normalen Herzen im Stande sein, die zu seiner vollständigen Füllung noch nothwendige Blutmenge von rechts her zu aspiriren, wenn die untere Hohlvene 
ihm nicht näher läge, als der rechte Vorhof. Das Blut müsste aber, entsprechend der anatomischen Anordnung der Theile ${ }^{1}$ ), aus dem rechten Vorhofe zurück in eine Ausweitung der unteren Hohlvene fliessen, bevor es in den linken Vorhof gelangen könnte. Deshalb also schon ist man gezwungen anzunehmen, dass der linke Ventrikel sich einen Zuschuss aus der unteren Hohlvene und nicht aus dem rechten Vorhofe nimmt, wenn die Lungenvenen, wie gewöhnlich, zu seiner Füllung nicht genügend Blut bringen.

In Anbetracht der Bedeutung, welche der mitgetheilte Fall für die eben berührten Fragen hat, hoffe ich auf die Verzeihung des geneigten Lesers dafür, dass ich seine Geduld mit nur einem Falle so lange in Anspruch genommen habe.

1) Siehe Wolff, Kilian und die übrigen obengenannten Autoren a. a. 0. 